\title{
Estimation of the minimum mean square error channel MMSE And energy efficiency management
}

\author{
Sara Riahi \\ Department of Computer Science, Chouaib Doukkali University, Faculty of Sciences , El Jadida, Morocco \\ riahisaraphd@gmail.com \\ Azzeddine Riahi \\ IMC Laboratory, Department of Physics, Chouaib Doukkali University, Faculty of Sciences, El Jadida, Morocco \\ riahikh@gmail.com
}

\begin{abstract}
In order to eliminate distortion due to signal propagation in a transmission channel, a multicarrier OFDM modulation scheme uses a channel estimation operation. Indeed, an estimate of the frequency response of the transmission channel is often necessary to achieve frequency equalization at the output of the FFT (Fast Fourier Transform). In the literature, most channel estimation techniques have been limited to exploiting the frequency correlation of the channel. Few of them have addressed the interests reflect the temporal correlation. Note that propagation in a mobile radio environment is characterized by frequency selectivity of the transmission channel, but also by mobility of transmitting / receiving equipment translated into time selectivity. This is expressed by a double time-frequency correlation of the frequency response of the transmission channel. In this paper, we propose to design a channel estimation algorithm, half-blind in a mobile-radio propagation environment. The initialization of this algorithm is done through information provided by the pilot OFDM symbols.
\end{abstract}

Keywords: Channel estimation, Quadrature Amplitude Modulation, MMSE, Wireless Systems, Energy Efficiency.

\section{Introduction:}

The OFDM / QAM are a good alternative to OFDM for transmitting high-speed signals in the current conditions of spectrum congestion. Indeed, the use of the cyclic prefix is not required in OFDM / QAM, increasing its spectral efficiency compared with OFDM. In addition, the symbols are not worn by cardinals sinuses, but by functions (called filters prototypes and rated $g$ ) greatly reducing out of band interference. Thus, in this paper, we use the frequency response given by [1]:

$$
G(f)=\sum_{k=-(K-1)}^{K-1} G_{k} \frac{\sin \left(\pi\left(f-\frac{k}{L_{f}}\right) L_{f}\right)}{L_{f}\left(\pi\left(f-\frac{k}{L_{f}}\right)\right)}
$$

Where $K$ is called the recovery factor (here $K=4$ ), $G_{0}=1$ and $G_{k}=G_{-k} . L_{f}$ is the length of the prototype filter. In Figure 1, it can be observed that the frequency response of the OFDM/QAM using the Bellanger filter has better frequency localization than the OFDM. However, this good spectral property can be obtained at the cost of a relaxation of the requirement of complex orthogonality, since the $g$ prototype filter is only orthogonal in the space of real $I R$. So instead of transmitting complex symbols at a rate $\tau_{0}$ as in OFDM, QAM symbols are real and transmitted at a rate $\tau_{0} / 2$. 


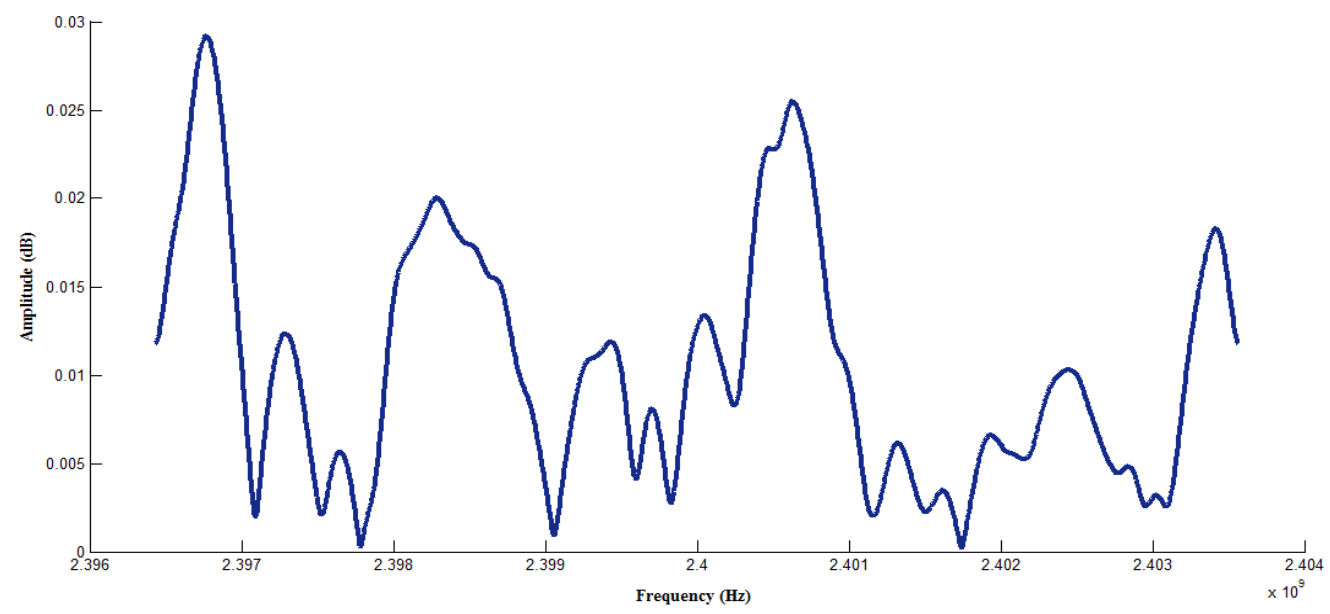

Fig 1: Frequency responses of OFDM

The OFDM/QAM signal received, after the serial /parallel conversion, the filtering in the polyphase network (defined by $g$ ) and the discrete Fourier transform (DFT) of size $M$, can be written [2]:

$$
y_{m, n}=H_{m, n} x_{m, n}+\underbrace{\sum_{(p, q) \in \Omega} H_{p, q} x_{p, q}<g>{ }_{m, n}^{p, q}+\omega_{m, n}}_{\mathrm{I}_{m, n}}
$$

Where the index $(m, n)$ indicates the position time-frequency, $x_{m, n}$ is the actual transmitted QAM symbol, $H_{m, n}$ is the channel frequency response, and $\omega_{m, n}$ is the additive Gaussian noise with zero mean and variance $\sigma^{2}$. The specificity of the OFDM / QAM modulation is found in the presence of the interference $I_{m, n}$ which is due to the filter $g$ and the channel [3]. The term $j<g>{ }_{m, n}^{p, q}$ in (2) is called "intrinsic interference" and is defined as the scalar product $<g_{m, n}, g_{p, q}>$, with $(p, q) \in \Omega$, where $\Omega$ is the set of frequency-time $(p, q)$ positions in the vicinity of $(m, n) \cdot j<g>{ }_{p, q}^{m, n}$ is a pure imaginary, so that if $H_{m, n}=1$, then we find the signal transmitted as $\hat{x}_{m, n}=\operatorname{Re}\left(y_{m, n}\right)$. However, the distortion of the complex channel coefficient $H_{m, n}$ induces a complex interference $I_{m, n}$. The channel must be estimated and reversed to recover the actual conditions of orthogonality. Many OFDM / QAM channel estimation methods have been proposed in the literature [4]. In this paper, we propose to study the MMSE estimator in OFDM/QAM. This estimate as a filter smoothing the frequency response of the channel, its use may be relevant in modulating considered where the received signal $y_{m, n}$ in (2) is subjected to distortion from noise and interference. In the remainder of the paper, the theoretical expression is derived for the MMSE estimator OFDM/QAM, and it shows that a 
simple formulation can be obtained, similar to that known in OFDM. The simulation results compare the MMSE estimator to OFDM/QAM and OFDM.

\section{MMSE estimators:}

\subsection{Theoretical expression of the estimator:}

To apply the MMSE estimation to the received signal, it is necessary to rewrite (2) in vector form:

$$
y_{n}=x_{n} H_{n}+I_{n}+w_{n}
$$

Where $y_{n}, H_{n}, I_{n}$ and $w_{n}$ are the vectors of size $M \times 1$ containing the samples defined in (2), and $x_{n}$ is the diagonal matrix whose elements are the transmitted symbols $x_{m, n}$. The developments given in [5] are used to give the general expression of the MMSE estimator, which aims at minimizing the cost function $J_{M M S E}$ defined by:

$$
J_{M M S E}=E\left\{\left\|H_{n}-\theta y_{n}\right\|_{F}^{2}\right\}
$$

Where $\theta$ is the matrix $M \times M$ whose coefficients are to be optimized. In (4), E\{.\} is the mathematical expectation and $\|.\|_{F}$ is the Frobenius norm. The cost function $J_{M M S E}$ can be reformulated by substituting (3) for (4) to obtain [6]:

$$
J_{\text {MMSE }}=\operatorname{tr}\left(R_{H H}+\theta\left(x_{n} R_{H H} x_{n}^{H}+R_{I I}+x_{n} R_{H I}+R_{I H} x_{n}^{H}+\sigma^{2} I_{d}\right) \theta^{H}-\theta\left(x_{n} R_{H H}+R_{I H}\right)-\left(R_{H H} x_{n}^{H}+R_{H I}\right) \theta^{H}\right)
$$

Where $R_{H H}, R_{I I}$ are respectively the matrix $M \times M$ of covariance of the $H_{n}$ channel and the interferences $I_{n}, R_{I H}$ and $R_{H I}$ the intercovariance matrix of $H_{n}$ and $I_{n}, I_{d}$ is the identity matrix

Using the rules of derivation of matrix in $C$ defined in [7], we find the minimum of $J_{M M S E}$ by solving:

$$
\frac{\partial J_{M M S E}}{\partial \theta^{*}}=\theta\left(x_{n} R_{H H} x_{n}^{H}+R_{I I}+x_{n} R_{H I}+R_{I H} x_{n}^{H}+\sigma^{2} I_{d}\right)-\left(R_{H H} x_{n}^{H}+R_{H I}\right)=0
$$

And we deduce [8]:

$$
\theta_{o p}=\left(R_{H H} x_{n}^{H}+R_{H I}\right) \times\left(x_{n} R_{H H} x_{n}^{H}+R_{I I}+x_{n} R_{H I}+R_{I H} x_{n}^{H}+\sigma^{2} I_{d}\right)^{-1}
$$

The MMSE estimator in OFDM / QAM is expressed from (8), factoring in $x_{n}(.) x_{n}^{H}$, as 


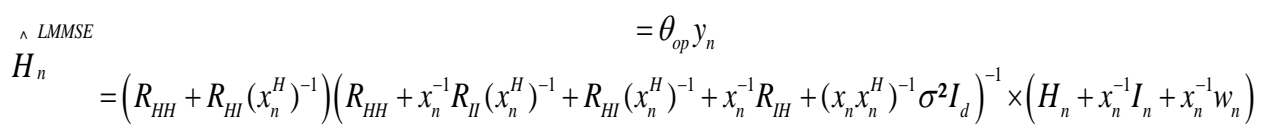

It has been raised in OFDM that the implementation of the MMSE estimator is limited by [9]:

- Its complexity (due to matrix inversions and multiplications).

- The absence of a priori knowledge of the covariance matrix of the $R_{H H}$ channel and the noise level $\sigma^{2}$.

This limitation is all the more true in OFDM/QAM since it appears in (8) that matrix $R_{I I}$ and $R_{H I}$ are required. Consequently, the MMSE implementation in (8) requires approximations [10]. This is discussed below.

\subsection{Simplified expression of the estimator:}

$$
\wedge \text { LMMSE }
$$

To simplify the expression of $H_{n} \quad$ given in (8), we can assume that [11]

- The source of the interfering symbols with $x_{m, n}$ is limited to $\Omega_{0}$ defined as the set of positions adjacent to $(m, n)$, $\Omega_{0}=\{(p, q) \mid p \in\{m-1, m, m+1\}, q \in\{n-1, n m+1\},(p, q) \neq(m, n)\} ;$

- The channel frequency response is assumed to be constant on the positions $(p, q) \in \Omega_{0}$, leading to the approximation $H_{p, q} \approx H_{m, n}$. These two assumptions allow rewrite (2) as [6]:

$$
\begin{aligned}
y_{m, n} & \approx H_{m, n}\left(x_{m, n}+x_{m, n}^{\prime}\right)+\omega_{m, n} \\
& =H_{m, n} d_{m, n}+\omega_{m, n}
\end{aligned}
$$

Where

$$
d_{m, n}=x_{m, n}+x_{m, n}^{\prime}=x_{m, n}+j \sum_{(p, q) \in \Omega_{0}} x_{p, q}<g>\begin{aligned}
& m, n \\
& p, q
\end{aligned}
$$

Such that (10) is very similar to the received signal in OFDM.

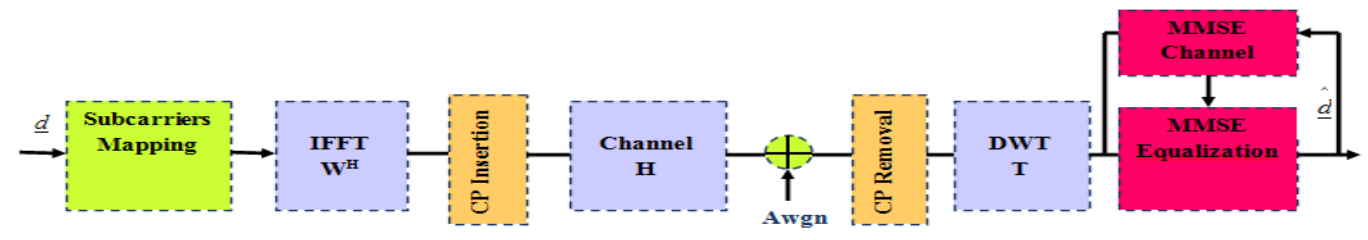

Fig 2: Block diagram for an improved joint MMSE channel estimation.

If $d_{m, n}$ is a pilot value with a known value, then it is possible, by analogy with the MMSE estimator OFDM give a simplified expression (8): 


$$
\hat{H}_{n}^{\text {LMMSE }}=\underbrace{R_{H H}\left(R_{H H}+\sigma^{2}\left(d_{n} d_{n}^{H}\right)^{-1}\right)^{-1}}_{K} \hat{H}_{n}
$$

Where $d_{n}$ is the diagonal $M \times M$ matrix containing the $d_{m, n}$ drivers, and $\hat{H}_{n}$ is the estimated channel response by [10]:

$$
\hat{H}_{n}=d_{n}^{-1} y_{n}
$$

Note that (13) is similar to the OFDM estimator using the least squares criterion, or least square (LS). However, it will be considered later that $x_{m, n}^{\prime}=0$ and therefore $d_{m, n}=x_{m, n}$ for all $0 \leq m \leq M-1$, so as to obtain an estimation in OFDM / QAM very similar to the LS estimator in OFDM. The method used is called ICM (for interference cancellation method) [11]. By $\wedge$ LMMSE

rewriting the MMSE estimator as in (12), it is possible to calculate $H_{n} \quad$ with reduced complexity using simplification methods such as the eigenvalue decomposition of $K$ and the low-order approximation of $R_{H H}$. Thus, the covariance matrix of the $R_{H H}$ channel is hermitian, so it is diagonalisable in an orthonormal basis such that $R_{H H}=U D_{R} U^{H}$, where $U$ is the unit matrix containing the eigenvectors of $R_{H H}$, and $D_{R}$ is the diagonal matrix containing the eigenvalues $\lambda_{0}, \lambda_{1}, \ldots, \lambda_{m}, \ldots, \lambda_{M-1}$ of $R_{H H}$. Moreover, assuming that the channel is a finite impulse response filter of length $L$, then it is also the rank $R_{H H}$ and therefore $\lambda_{L}=\lambda_{L+1}=\ldots .=\lambda_{M-1}=0$.

In practice, the non-zero eigenvalues of $R_{H H}$ are rarely known a priori [12]. However, $R_{H H}$

can be approximated by a well-known $\tilde{R}_{H H}$ matrix whose spectrum is imposed (corresponding to the power profile of the channel). Without loss of generality, we consider a constant profile, so that the decomposition into eigenvalues (also known) gives $\tilde{R}_{H H}=\tilde{U} \tilde{D}_{R} \tilde{U}^{H}$, where the eigenvalues $\tilde{\lambda}_{m}$ on the diagonal of $\tilde{D}_{R}$ are equal to $\frac{1}{L}$ if $0 \leq m \leq \tilde{L}-1$, and 0 otherwise. As noted in [5], the quality of the MMSE estimator using the low rank approximation is guaranteed for $\tilde{L} \geq L$.

We assume this condition fulfilled in the following, and we can rewrite (12) using $\tilde{R}_{H H}$ instead of $R_{H H}$ and we define $\tilde{K}=\tilde{R}_{H H}\left(\tilde{R}_{H H}+\sigma^{2}\left(d_{n} d_{n}^{H}\right)^{-1}\right)^{-1}$. Since $\sigma^{2}\left(d_{n} d_{n}^{H}\right)^{-1}$ is a diagonal matrix, then $\tilde{K}$ is diagonalisable in the same basis as $\tilde{R}_{H H}$, and we can rewrite the MMSE estimator as $\hat{H}_{n}^{\text {LMMSE }}=\tilde{U} \tilde{D}_{K} \tilde{U}^{H} \hat{H}_{n}$, where $\tilde{D}_{K}$ is the eigenvalue decomposition of $\tilde{K}$ such that its elements are defined by [10]: 


$$
\tilde{\mu}_{m}=\left\{\begin{array}{ccc}
\frac{\tilde{\lambda}_{m}}{\tilde{\lambda}_{m}+\frac{\sigma^{2}}{\left|d_{m}\right|^{2}}} & \text { if } & m=0,1, \ldots ., \tilde{L}-1 \\
0 & & m=\tilde{L}, \tilde{L}+1, \ldots ., M-1
\end{array}\right.
$$

Thus, it is possible to use the MMSE estimator for OFDM / QAM modulation without prior knowledge of $R_{H H}$, and reducing the complexity (given in number of multiplications) of

$O\left(2 M^{3}\right)$ in (12) to $O\left(\tilde{L} M^{2}+L \tilde{M}\right)$ using (14).

\section{Simulation and results analysis:}

In this part, we simulate the validity of the simplified MMSE estimator in OFDM/QAM and compare its performance (Figure 3). For this, we use the parameters of the standard LTE (Long Term Evolution).

The actual QAM symbols are chosen in $[-1,+1]$ (equivalently, the symbols for the OFDM signal come from a QAM), and no channel code is used. The transmission channel is a Gaussian channel, and we consider a simple ZF (zero forcing) equalization on each carrier.

Figure 4 shows the estimation MSE obtained for LS (in OFDM), and simplified MMSE using the OFDM and OFDM/QAM eigenvalue decomposition, as a function of Eb/N0 (dB). The curves are averages obtained on several random realizations of the channel. There is a high gain between the MMSE estimate and the LS and MRC estimators, reflecting the "smoothing" effect of MMSE on the frequency response of the channel.

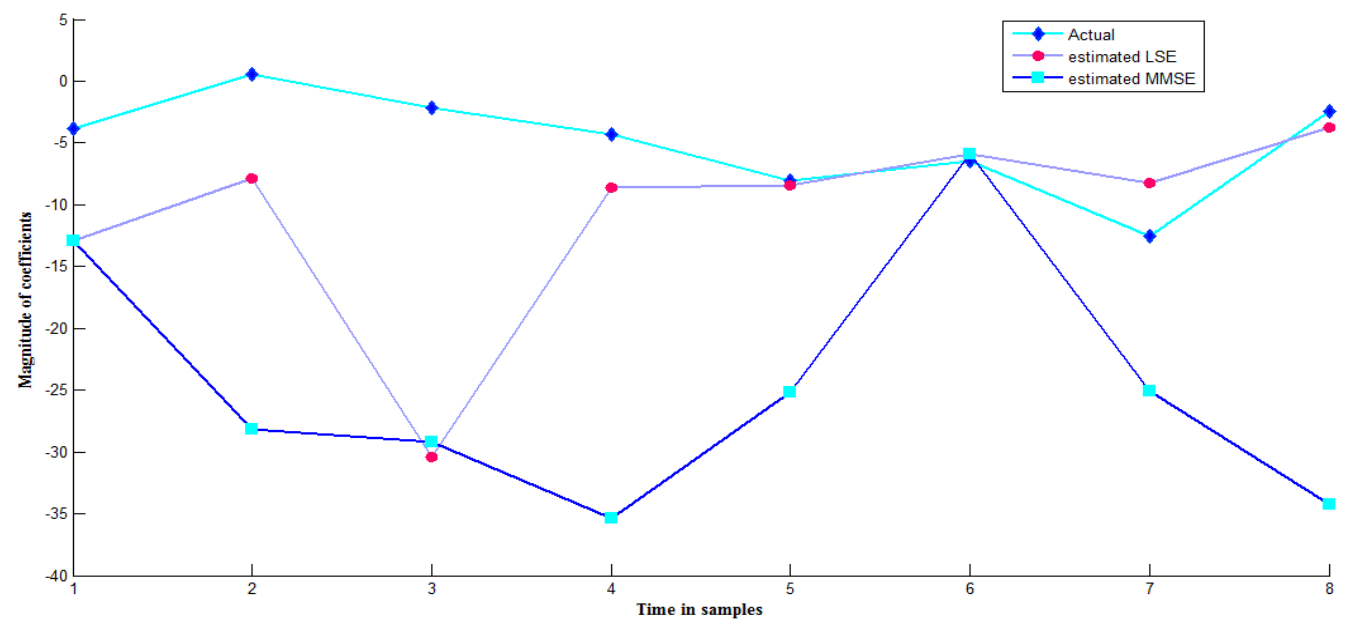

Fig 3: Channel estimation using LS and MMSE estimators

This effect remains valid OFDM / QAM. However, there is a MSE threshold is reached in OFDM/QAM for MRC and MMSE, due to interference inherent to this type of modulation. Figure 5 shows the bit error rate (BER) as a function of Eb / N0 (dB) obtained for the same estimators as before. Moreover, the curves of a transmission in a Gaussian channel (denoted by AWGN, additive white Gaussian noise for) and perfect estimation are displayed as references. A gain obtained for the MMSE estimators compared to the LS and ZF estimators is observed, confirming the good performance of MMSE observed in Figure 5. It should be noted, however, 
that the curve corresponding to MRC has a lower slope than LS from Eb / N0 $=15 \mathrm{~dB}$, due to the presence of intrinsic interference in OFDM/QAM.

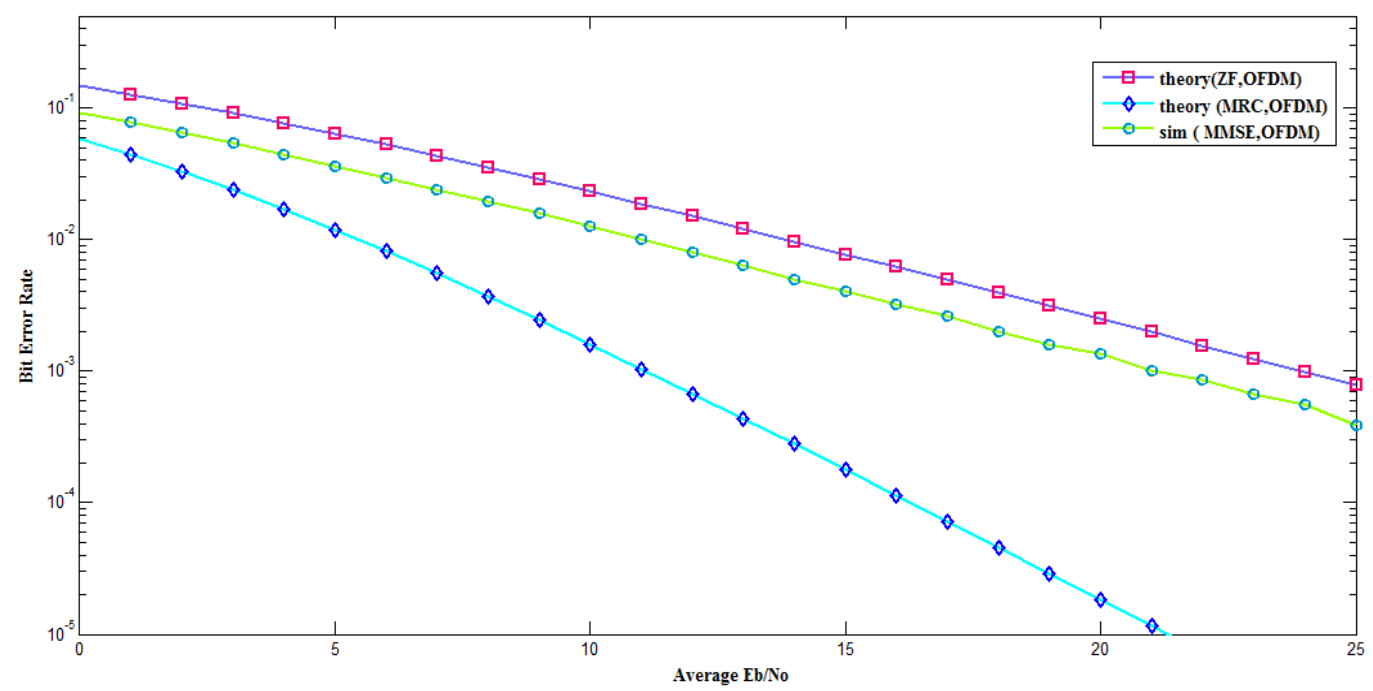

Fig 4: BER for QAM modulation, MMSE equalizer, AWGN channel

These interferences are embedded in noise for low values of $\mathrm{Eb} / \mathrm{N} 0$, but appear for low noise values. The same phenomenon is observed by comparing the curves of the MMSE estimator in OFDM and OFDM/QAM. However, we note that the loss of OFDM/QAM over OFDM is only $0.2 \mathrm{~dB}$ at $\mathrm{Eb} / \mathrm{N} 0=25 \mathrm{~dB}$. For lower values of $\mathrm{Eb} / \mathrm{N} 0$, the two curves are combined, and are only $0.1 \mathrm{~dB}$ of the perfect estimate.

These results show that despite the complex formulation of the MMSE estimator in OFDM/QAM in (8), it is possible to obtain a simplified version of the estimator while keeping a performance close to the perfect estimate.

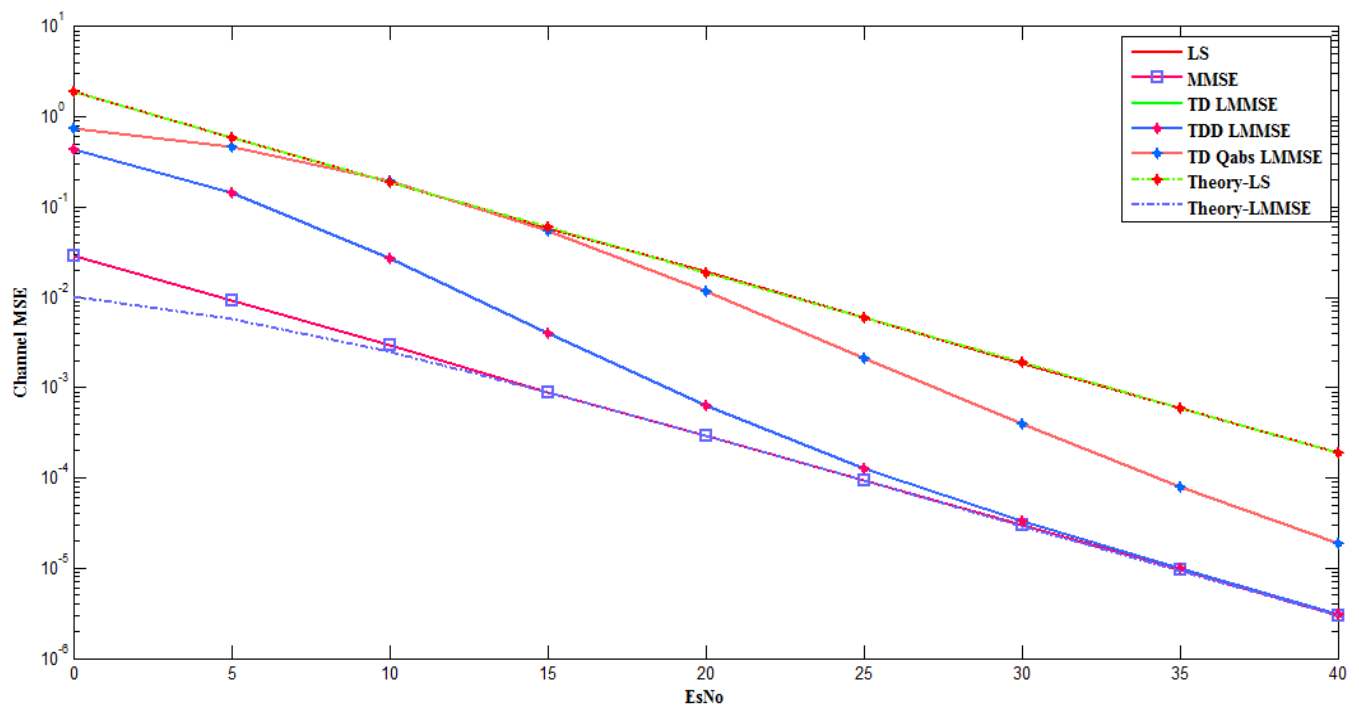

Fig 5: Comparison of different channel estimation techniques with MMSE

In addition, the BER of the MMSE in OFDM/QAM is very similar to that obtained in OFDM. This result is even more interesting that in general, the spectral efficiency of OFDM/QAM (not 
using a cyclic prefix) is greater than the OFDM. The analysis of the curves obtained shows that the estimation studied technique introduces improvements in performance of the channel estimation.

\section{Conclusion:}

In this paper, we introduced the channel estimator based on the criterion of the mean square error (MMSE) in OFDM with the quadrature amplitude modulation. The theoretical expression of the estimator has been proposed, and it shows that it is not applicable, due in particular to its excessive complexity. However, approximations enable a new formulation similar to that known in OFDM. Thus, it is possible to use simplification methods of the MMSE channel estimator, such as the eigenvalue decomposition and the low rank approximation. Simulation results have shown that the MMSE channel estimator in OFDM / QAM is able to achieve the same performance in OFDM, for better spectral efficiency.

\section{Acknowledgment:}

We would like to thank the CNRST of Morocco (I 012/004) for support.

\section{References:}

[1] M.Tuchler, C.Andrew, Singer and R .Koetter, ,Minimum Mean Squared Error equalization using priori information ,'IEEE Trans, on signal process., vol. 50,no. 3, pp 673-683 mar.2002

[2] Sara RIAHI, Azzeddine RIAHI , "Applications of game theory to optimization of resource allocation of wireless systems: algorithmic solution " ,International Journal of Online Engineering (iJOE), ISSN: 2626-8493, PP. 5-25,Vol 14, No 12 (2018).

[3] X.-B. Liang, "Orthogonal designs with maximal rates", IEEE Trans.Inform.Theory, vol. 49, no. 10, pp. 2468-2503,. 2003.

[4] M. K. Karakayali, G. J. Foschini, R. A. Valenzuela, "Network coordination for spectrally efficient communications in cellular systems", IEEE Wireless Commun. Mag., vol. 13, no. 4, pp. 56-61, Aug. 2006

[5] Sara RIAHI, Azzeddine RIAHI, " Game theory for resource sharing in large distributed systems ", International Journal of Electrical and Computer Engineering, IJECE, Vol. 9, No. 2, April 2019, pp. 1249 1257 ISSN: 2088-8708, DOI: 10.11591/ijece.v9i2.pp.1249-1257, IJECE.

[6] Jittra Jootar, James R. Zeidler, John G. Proakis, "On the Performance of Closed-Loop Transmit Diversity with Noisy Channel Estimates and Finite-Depth Interleaved Convolutional Codes", Communications IEEE Transactions on, vol. 56, 2008, ISSN 0090-6778.

[7] K. Cho, D. Yoon, "On the general BER expression of one- and two-dimensional amplitude modulations", IEEE Trans. Commun., vol. 50, pp. 1074-1080, July 2002.

[8] Y. Yang, Q. Huang, Z. Zhang, "Vehicular Technology Conference 2009", An Efficient Joint Timing and Frequency Offset Estimation for OFDM Systems, pp. 1-5.

[9] Mohammed Ali Mohammed MOQBEL1 , Wangdong2 , Al-marhabi Zaid Ali, "MIMO Channel Estimation Using the LS and MMSE Algorithm ",IOSR Journal of Electronics and Communication Engineering (IOSR-JECE) e-ISSN: 2278-2834.Volume 12, Issue 1, Ver. II (Jan.-Feb. 2017), PP 13-22

[10] Ajay Bahadur Singh1, Vivek Kumar Gupta, "Performance Evaluation of MMSE and LS Channel Estimation in OFDM System ",International Journal of Engineering Trends and Technology (IJETT) Volume 15 Number 1 - Sep 2014

[11] M.-X. Chang, "A new derivation of least-squares-fitting principle for OFDM channel estimation", IEEE Trans. Wireless Commun., vol. 5, no. 4, pp. 726-731, Apr. 2006.

[12] Akash Kumar Shrivas, "A Comparative Analysis of LS and MMSE Channel Estimation Techniques for MIMOOFDM System ",IJIRST -International Journal for Innovative Research in Science \& Technology| Volume 1 | Issue 8 | January 2015 ISSN (online): 2349-6010 